\title{
A Novel Method for Incorporation of Micron-Sized SiC Particles into Molten Pure Aluminum Utilizing a Co Coating
}

\author{
M. MOHAMMADPOUR, R. AZARI KHOSROSHAHI, \\ R. TAHERZADEH MOUSAVIAN, and D. BRABAZON
}

\begin{abstract}
Ceramic particles typically do not have sufficiently high wettability by molten metal for effective bonding during metal matrix composite fabrication. In this study, a novel method has been used to overcome this drawback. Micron-sized SiC particles were coated by a cobalt metallic layer using an electroless deposition method. A layer of cobalt on the $\mathrm{SiC}$ particles was produced prior to incorporation in molten pure aluminum in order to improve the injected particle bonding with the matrix. For comparison, magnesium was added to the melt in separate experiments as a wetting agent to assess which method was more effective for particle incorporation. It was found that both of these methods were more effective as regard ceramic particulate incorporation compared with samples produced with as-received $\mathrm{SiC}$ particles injected into the pure aluminum matrix. SEM images indicated that cobalt coating of the particles was more effective than magnesium for incorporation of fine $\mathrm{SiC}$ particles (below $30 \mu \mathrm{m}$ ), while totally the incorporation percentage of the particles was higher for a sample in which $\mathrm{Mg}$ was added as a wetting agent. In addition, microhardness tests revealed that the cobalt coating leads to the fabrication of a harder composite due to increased amount of ceramic incorporation, ceramic-matrix bonding, and possibly also to formation of Al-Co intermetallic phases.
\end{abstract}

DOI: $10.1007 / \mathrm{s} 11663-014-0186-9$

(C) The Minerals, Metals \& Materials Society and ASM International 2014

\section{INTRODUCTION}

Aluminum metal matrix composites (AMMCs) have gained significant attention in recent years. This is primarily due to their lightweight, low coefficient of thermal expansion (CTE), good machinability character, and improved mechanical properties, such as increased 0.2 pct YS, UTS, and hardness. Due to these advantages, they are used in aerospace industries (airframe and aerospace components), automobile industries (engine pistons), and electronic components. ${ }^{[1-8]}$

Many techniques have been developed for producing particulate-reinforced AMMCs, such as stir casting, squeeze casting, ${ }^{[3-18]}$ and powder metallurgy. ${ }^{[19-21]}$ Although each of these methods has its own advantages and disadvantages, they are all relatively expensive. Nowadays, researchers are focusing on developing lowcost methods of producing composites. Stir casting (vortex technique) is generally accepted commercially as a low-cost method. Its advantages lie in its simplicity,

M. MOHAMMADPOUR, M.Sc Graduate Student and R. AZARI KHOSROSHAHI, Associate Professor, President, are with the Faculty of Materials Engineering, Sahand University of Technology, Tabriz, Iran. R. TAHERZADEH MOUSAVIAN, Lecturer, is with the Department of Metallurgy, Zanjan Branch, Islamic Azad University, Zanjan, Iran. Contact e-mails: rtaher1898@gmail.com, r_taherzadeh@ sut.ac.ir D. BRABAZON, Associate Professor, is with the Advanced Processing Technology Research Centre, School of Mechanical \& Manufacturing Engineering, Dublin City University, Dublin 9, Ireland. Manuscript submitted May 16, 2014.

Article published online September 12, 2014. flexibility, and applicability to large volume production. This process is the most economical of all the available routes for AMMCs production and allows very largesized components to be fabricated. However, methods of achieving the following in stir casting are mostly to be considered: (i) no adverse chemical reaction between the reinforcement material and matrix alloy, (ii) no or very low porosity in the cast AMMCs, (iii) wettability between the two main phases, and (iv) achieving a uniform distribution of the reinforcement material. ${ }^{[3-18]}$

Some of the methods used to achieve these goals during stir casting of aluminum matrix composites are the modification of the alloy composition, coating or specific treatments to the reinforcements, and control of the process parameters (stirring temperature and time, etc.). ${ }^{[11-14]}$ Among these, coating of the reinforcement is a successful technique used to prevent adverse interfacial reaction and promote wetting of the particles by aluminum through increasing the overall surface energy of the solid. Metallic coatings of nickel or copper have been widely used to improve the wettability of carbon fibres and ceramic particles by molten aluminum alloys. ${ }^{[22-29]}$ To the best of our knowledge, no attempt has been made to coat ceramic particles with cobalt using an ED method.

In this study, micron-sized $\mathrm{SiC}$ particles were coated with cobalt using the ED method. The coated particles were then incorporated into the molten pure aluminum to assess the effects of this process on the ceramic incorporation. For comparison to these composites, another sample type was fabricated, in which established wetting agent magnesium was added. The aim of this study was to compare the effect of cobalt coating using a 
low-cost ED method with another low-cost simple method in which established wetting agent magnesium was added. The particulate incorporation percentage and fabricated composite microstructures after solidification were compared.

\section{EXPERIMENTAL PROCEDURES}

Aluminum ingot with 99.8 in wt pet commercial purity was used as a matrix. The chemical composition of the used ingot obtained using a M5000 optical emission spectrometer is given in Table I. As can be seen, the amount of $\mathrm{Si}$ and $\mathrm{Fe}$ was negligible.

Micron-sized SiC particles with an average particle size of $80 \mu \mathrm{m}$ and 99.9 pct purity were supplied (Shanghai Dinghan Chemical Co., Ltd. China) as the reinforcement of metal matrix composite. The morphology of the silicon carbide particles is shown in Figure 1. Microstructural investigations were performed using two types of scanning electron microscope (SEM, Cam Scan Mv2300, equipped with EDAX analysis and SEM, KYK Y-EM3200).

Figure 2 presents the steps used to coat the $\mathrm{SiC}$ particles. The powders were first pre-treated using the three processes of etching, sensitization, and activation.

Table II summarizes the details of the $\mathrm{SiC}$ powder pre-treatment processes and chemicals used. Also, Table III shows the chemicals which were used for cobalt electroless coating as well as their concentrations. Also, the amounts of magnetic stirring, $\mathrm{pH}$, temperature, and time are reported in this table.

Three sample types were fabricated in this study for comparison of their effects on reinforcement incorporation. In one type, the $\mathrm{SiC}$ was injected into the aluminum without the use of an additional wetting aid; in a second type, magnesium was added to the melt before $\mathrm{SiC}$ particle injection; and in the third type, cobalt-coated $\mathrm{SiC}$ particles were injected into the molten aluminum. Table IV summaries these sample preparation methods. The same as-received SiC powders, with their morphology shown in Figure 1, were used for cobalt coating.

One gram of reinforcement powder was encapsulated in an aluminum foil packet for insertion into the molten aluminum in order to fabricate a composite with 3 wt pet $\mathrm{SiC}$ as reinforcement. The pure aluminum was heated to $953 \mathrm{~K}\left(680^{\circ} \mathrm{C}\right)$ within a bottom-pouring furnace. A graphite stirrer was placed below the surface of melt and rotated with a speed of $500 \mathrm{rpm}$, and simultaneously, argon gas with a high purity was used as a protective shroud on the melt surface. Figure 3(a) shows the schematic of the vortex casting setup, and Figure 3(b) shows a low-carbon steel mold into which the samples were cast. The packets were added to the vortex center, and the stirring was continued after this for 6 minutes. The composite slurry was poured into the preheated low-carbon steel mold [at $723 \mathrm{~K}\left(450{ }^{\circ} \mathrm{C}\right)$ ]. The yellow-colored marked point in Figure 3(b) is the location from which samples were taken for microstructural characterization.

Microhardness tests were conducted according to ASTM E384 using an applied load of $25 \mathrm{~g}$ for a

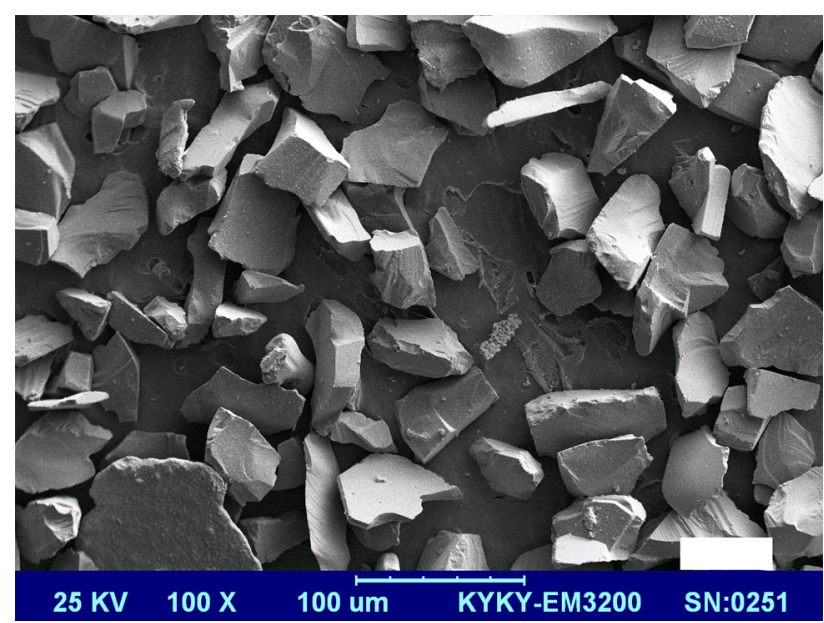

Fig. 1-The morphology of $\mathrm{SiC}$ particles which were used as reinforcement.

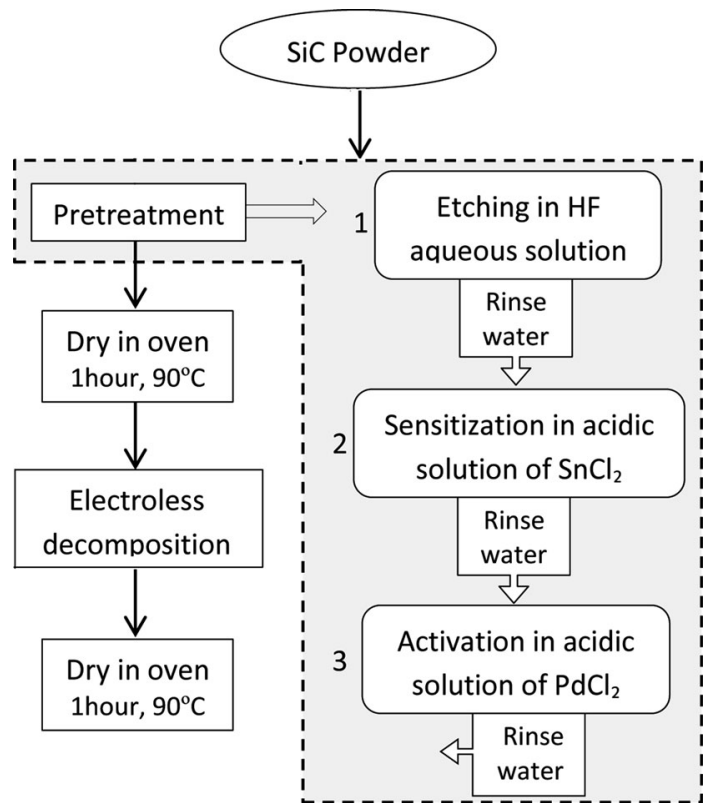

Fig. 2-Flow chart of preparation procedure used for production of the Co-coated $\mathrm{SiC}$ particles.

Table I. The Chemical Composition (in Weight Percent) of Pure Aluminum Used in This Study

\begin{tabular}{lcccccccc}
\hline Al pct & Si pct & Fe pct & Cu pct & Mn pct & Zn pct & Ni pct & Pb pct & Sn pct \\
\hline $99.8 \mathrm{~min}$ & 0.1 & 0.04 & 0.01 & 0.01 & 0.02 & 0.01 & 0.005 & 0.004 \\
\hline
\end{tabular}


Table II. Details of the SiC Powder Pre-Treatment Processes and Chemicals Used

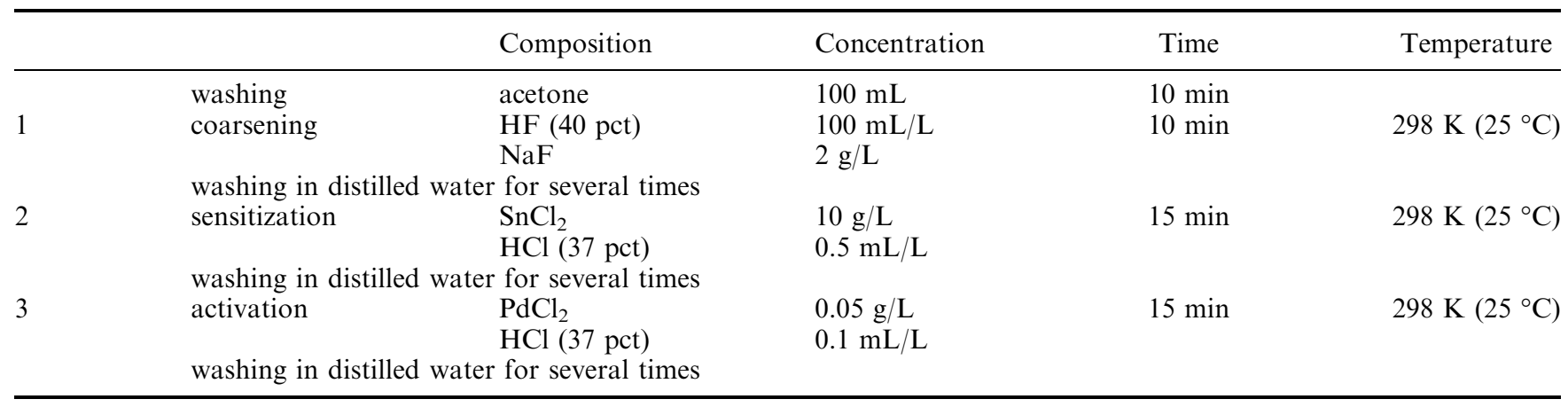

Table III. Composition of Bath Used for Electroless Deposition of Co Coating on SiC Particles as Well as the Parameters of Coating

\begin{tabular}{llll}
\hline Role in Bath & & Composition & \\
\hline Main Salt & Cobalt Sulfate & $\mathrm{CoSO}_{4} \cdot 7 \mathrm{H}_{2} \mathrm{O}$ & $25 \mathrm{~g} / \mathrm{L}$ \\
Reducing agent & sodium hypophosphite & $\mathrm{NaH}_{2} \mathrm{PO}_{2} \cdot \mathrm{H}_{2} \mathrm{O}$ & $25 \mathrm{~g} / \mathrm{L}$ \\
Complexing agent & tri-sodium citrate & $\mathrm{C}_{6} \mathrm{H}_{5} \mathrm{Na}_{3} \mathrm{O}_{7} \cdot 2 \mathrm{H}_{2} \mathrm{O}$ & $50 \mathrm{~g} / \mathrm{L}$ \\
Buffering agent & acid boric & $\mathrm{H}_{3} \mathrm{BO}_{3}$ & $25 \mathrm{~g} / \mathrm{L}$ \\
pH adjuster & sodium hydroxide & $\mathrm{NaOH}$ & to adjust $\mathrm{pH}$ \\
SiC powder & & & $0.5 \mathrm{~g} / 200 \mathrm{~mL}$ \\
Operation & magnetic stirring & & $400 \mathrm{rpm}$ \\
& temperature & & $70{ }^{\circ} \mathrm{C}$ \\
& pH & & 9 \\
& time & & $115 \mathrm{~min}:$ to complete reaction \\
\hline
\end{tabular}

Table IV. Characteristics of the Samples Fabricated in this Study

\begin{tabular}{lc}
\hline Samples & Characteristics of Matrix and Reinforcement \\
\hline $\mathrm{S}_{1}$ & pure Al as matrix and as-received SiC powders \\
& as reinforcement \\
$\mathrm{S}_{2}$ & Al-1 wt pct $\mathrm{Mg}$ as matrix and as-received $\mathrm{SiC}$ \\
& powders as reinforcement \\
$\mathrm{S}_{3}$ & pure $\mathrm{Al}$ as matrix and Co-coated SiC powders \\
& as reinforcement \\
\hline
\end{tabular}

15 seconds duration. At least 10 such measurements were taken from each composite.

\section{RESULTS AND DISCUSSION}

Figure 4 shows the microstructure of sample $S_{1}$, indicating that a very low amount of ceramic particles were incorporated into the molten metal. This figure shows that the $80-\mu \mathrm{m} \mathrm{SiC} \mathrm{particles} \mathrm{did} \mathrm{not} \mathrm{have} \mathrm{enough}$ wettability to be well incorporated into the molten aluminum.

The addition of alloying elements to the aluminum matrix has been reported to be a suitable method for improving the wettability of ceramic by the metal. ${ }^{[13]} \mathrm{It}$ was shown in our previous study ${ }^{[30]}$ that magnesium is the best alloying element for incorporation of micronsized $\mathrm{SiC}$ particles into the melt of pure aluminum.
Figure 5 shows the microstructure of sample $S_{2}$, in which magnesium was added in chip form before ceramic addition.

Figure 5 reveals that magnesium is very effective in improving the wettability of ceramic particles by the molten aluminum compared to the previous sample. Agglomeration occurred in some parts, while in general a good distribution of ceramic particles was revealed. Some porosities were detected around the particles, which were probably caused by solidification shrinkage between ceramic powders and the aluminum matrix. Some gas pores could also be seen in the matrix due to gas evolution and entrapment during casting. It was also found that a void formed between some agglomerated particles where reduced permeability would be expected. Most of the ceramic particles appeared to be larger than $50 \mu \mathrm{m}$ in mean diameter size. Yellow-colored rectangles in Figure 5 show the particles which are smaller than the mean diameter size. Figure 1 shows a considerable presence of the ceramic particles with a mean diameter size below $50 \mu \mathrm{m}$. It seems that magnesium was more effective to incorporate larger $\mathrm{SiC}$ particles or that the smaller particles were segregated. In conclusion, it should be noted that magnesium addition is a simple low-cost method, which can be used for fabrication of cast aluminum matrix composites reinforced with micron-sized ceramic particles.

Metallic coating of ceramic particles forms a layer around the particles, which can increase the wettability of particle by the molten metal phase. ED method is a 


\section{Argon gas inlet}

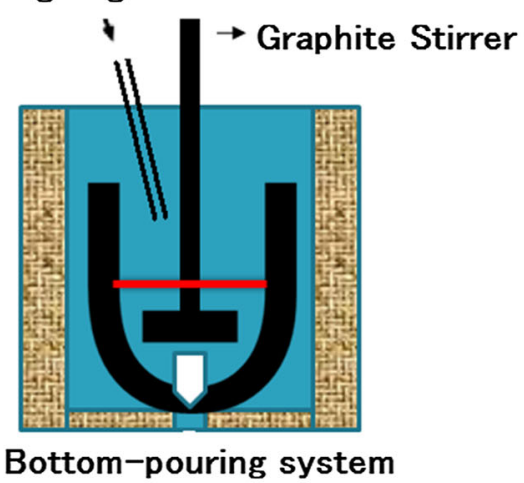

(a)

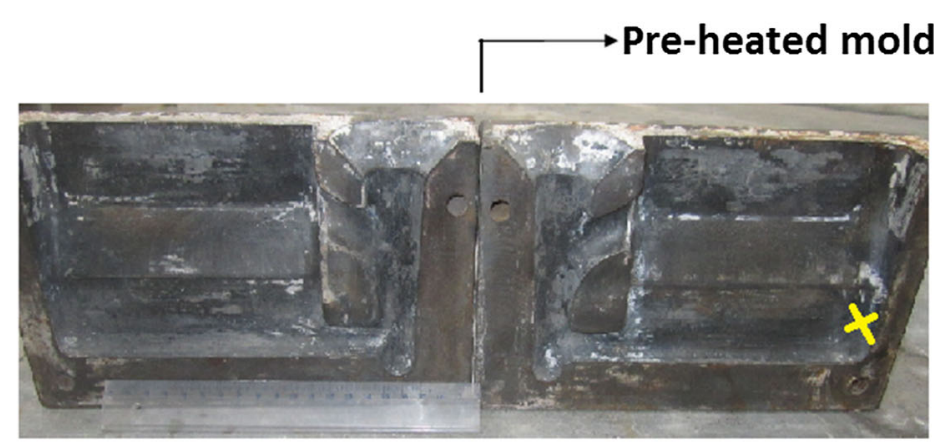

(b)

Fig. 3-The experimental setup used in this showing the (a) schematic of the vortex casting setup and (b) the low-carbon steel mold for casting into.

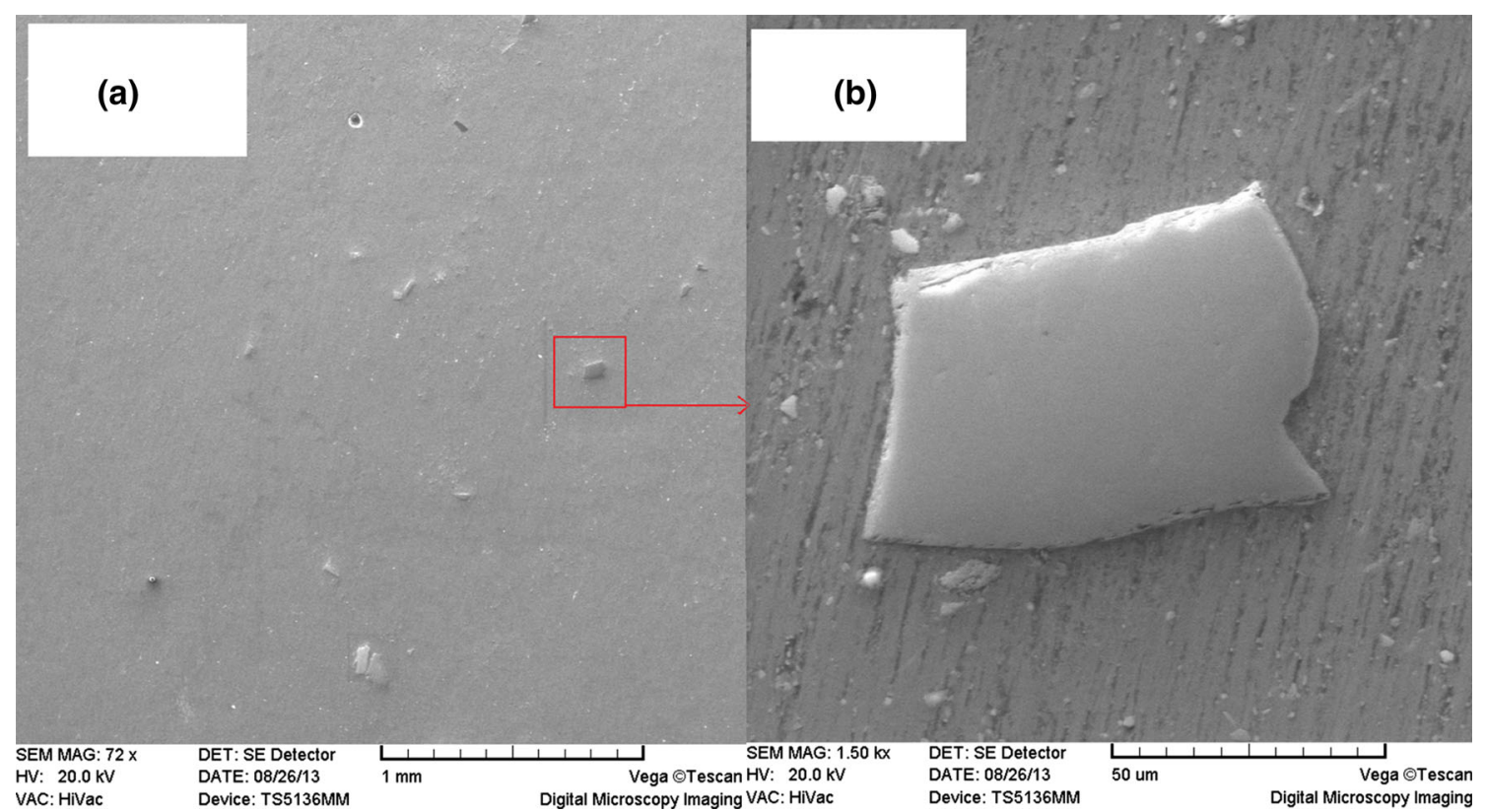

Fig. 4 - (a) Low- and (b) high-magnification SEM microstructures of sample $\mathrm{S}_{1}$ after vortex casting.

simple low-cost route which could fabricate a core ceramic-metallic shell structure to be used for industrial applications. Figure 6 shows the morphology of $\mathrm{SiC}$ powders coated by cobalt. As it can be seen, both fine and coarse powders were uniformly coated by cobalt, and it seems that the coating layer was sufficiently thick. It is very important to note that various $\mathrm{pH}$ and bath temperatures were investigated to obtain this good quality of coating. It was found in our previous studies that $\mathrm{pH} 9$ and bath temperature of $343 \mathrm{~K}\left(70^{\circ} \mathrm{C}\right)$ (Table I) produced the best conditions for this cobalt coating. The coating layer seems to be well adhered (Figure 6). Line EDAX analysis, shown in Figure 6, indicated that the intensity of $\mathrm{Co}$ and $\mathrm{P}$ (phosphorus comes from sodium hypophosphite, Table III) at the surface was considerable compared to the intensity of silicon at coated parts. However, it could be seen that in some parts, the SiC powders were not fully coated after
ED process, and the surface intensity of silicon was higher in these regions as expected. It should be noted that carbon has a small atomic radius and therefore, its detection during EDAX analysis is not accurate.

These coated ceramic particles were encapsulated in the aluminum foil and injected into the molten pure aluminum, as described earlier, to form sample $\mathrm{S}_{3}$. Figure 7 shows an SEM image of this sample microstructure obtained after vortex casting. It is evident in this figure that the number of coated $\mathrm{SiC}$ particles is appreciable. An important point to note from this figure is the presence of small $\mathrm{SiC}$ particles below $30 \mu \mathrm{m}$. It seems that cobalt coating formed via the ED process would be a suitable method for the incorporation of fine micron ceramic particles. For sample $\mathrm{S}_{3}$, a composite with a more uniform distribution of ceramic particles was fabricated compared to the previous samples $S_{1}$ and $\mathrm{S}_{2}$. The uniform distribution of the ceramic particles 


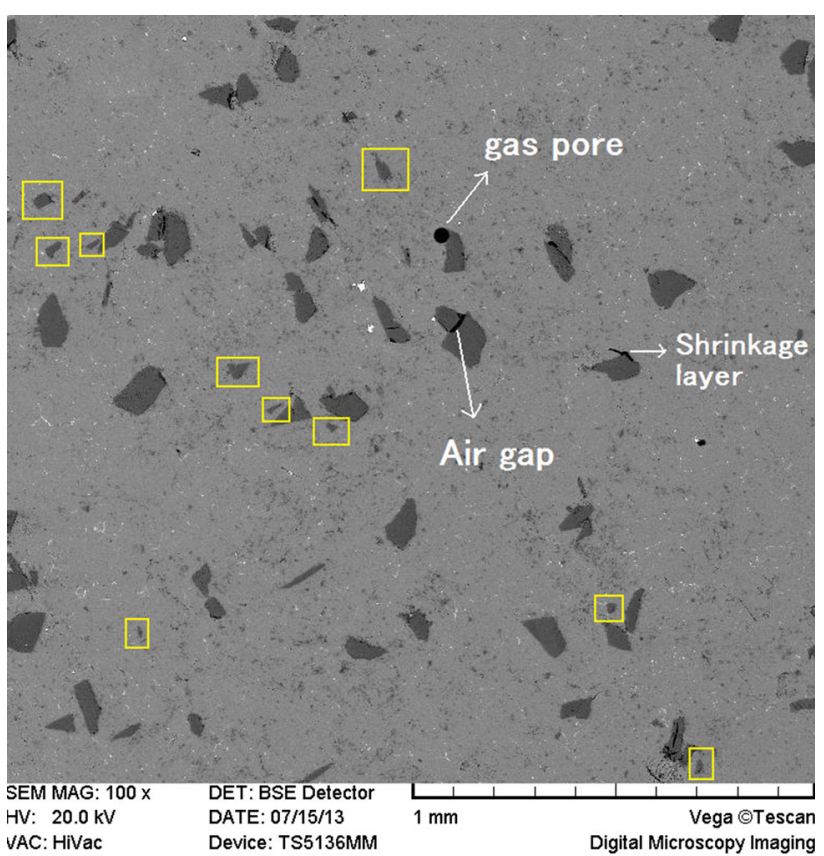

Fig. 5-SEM microstructure of sample $\mathrm{S}_{2}$ containing magnesium as a wetting agent. indicated in Figure 7 shows that this method might be also useful to avoid ceramic particle agglomeration.

In addition to the typical difficulty of obtaining good wettability, adverse chemical reaction between aluminum and $\mathrm{SiC}$ occurs at temperatures above $953 \mathrm{~K}$ $\left(680{ }^{\circ} \mathrm{C}\right)$, leading to the production of detrimental $\mathrm{Al}_{4} \mathrm{C}_{3}$ phase. ${ }^{[8,12,22,23]}$ The cobalt metallic coating can largely avoid such reaction if it is present on the surface of the particles. Figure 8 shows a high-magnification SEM micrograph of a $\mathrm{SiC}$ particle where a fingerprint-like structure was formed around the $\mathrm{SiC}$ particles (white colored parts). Both point and line EDAX analysis indicated that this structure is due to the cobalt coating where dissolution of the cobalt coating has occurred in the matrix during stirring at $953 \mathrm{~K}\left(680{ }^{\circ} \mathrm{C}\right)$. Point EDAX analysis (at the locations of the red-colored square) shows the presence of about 14 pct atomic cobalt in this part. It is important to note that Al-Co binary system has three important intermetallic compounds close to the aluminum side. ${ }^{[31]}$ Based on the AlCo phase diagram, $\mathrm{Al}_{9} \mathrm{Co}_{2}, \mathrm{Al}_{13} \mathrm{Co}_{4}$, and $\mathrm{Al}_{3} \mathrm{Co}$ are the phases which could be formed between cobalt and molten aluminum. It seems that atomic percent of cobalt vs aluminum obtained in this study is close to $\mathrm{Al}_{9} \mathrm{Co}_{2}$ phase with monoclinic structure. ${ }^{[31]}$ However, microhardness test results indicated that even this fingerprint-
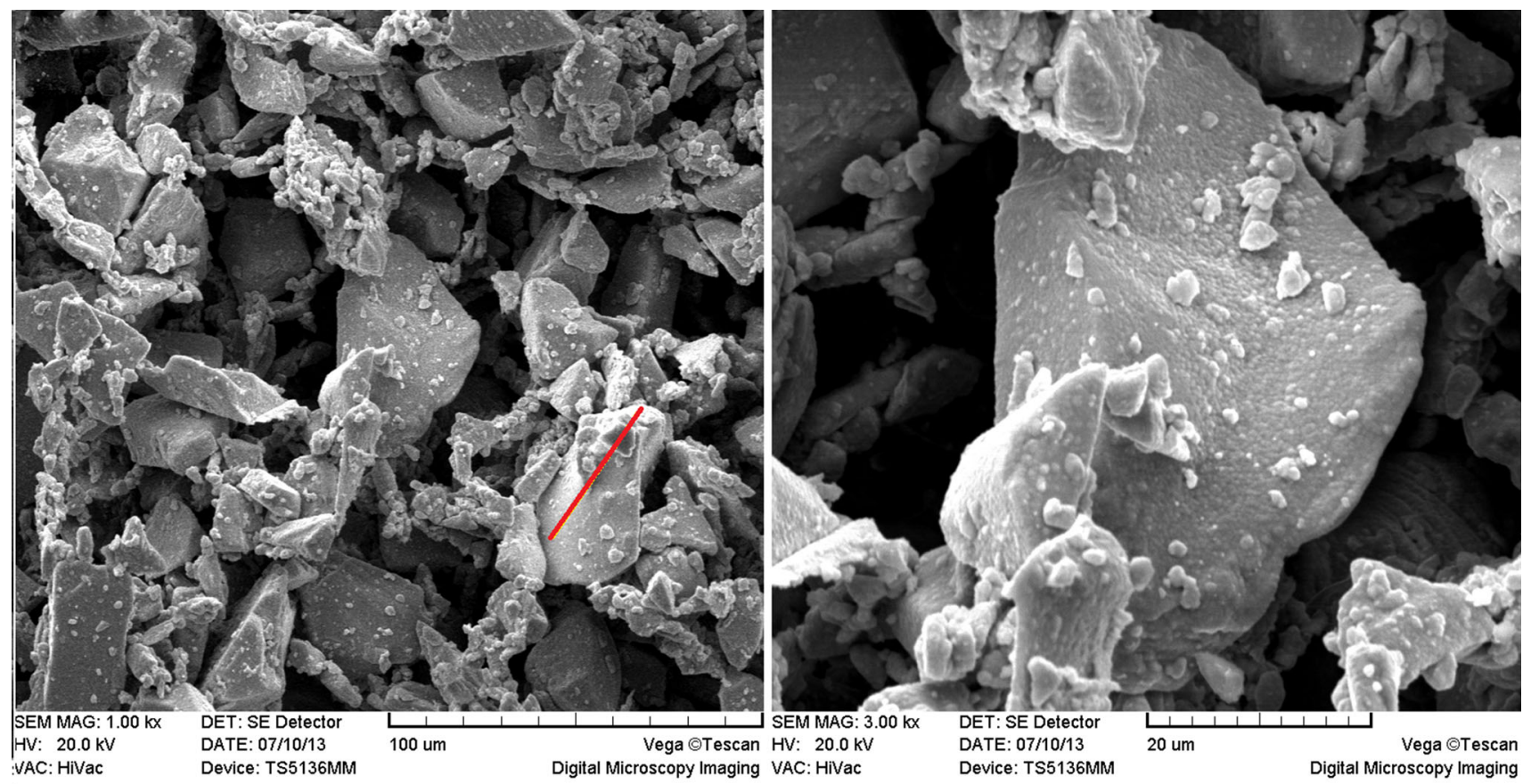

VAC: Hivac

Device: TS5136MM

Digital Microscopy Imaging VAC: HiVac
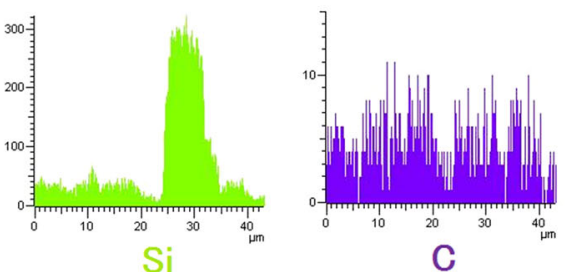

Fig. 6-The morphology of Co-coated SiC particles after ED process as well as line EDAX analysis of the powder surface. 
like structure of cobalt would be effective for improving the mechanical properties of the composite.

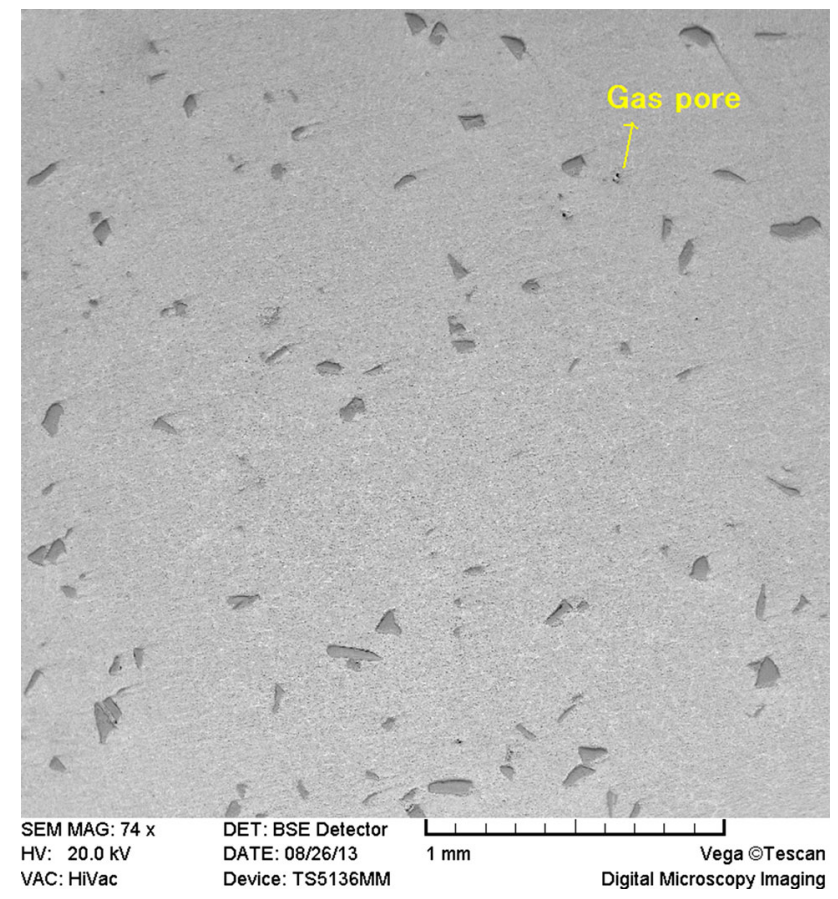

Fig. 7-SEM image of sample $S_{3}$ after vortex casting.
Figure 9 shows the effect of $\mathrm{Mg}$ addition and cobalt coating on the ceramic incorporation percent. As it can be seen from this figure, both these methods are able to highly incorporate the ceramic particles. The incorporation percentage of sample $S_{2}$ is higher than that of sample $\mathrm{S}_{3}$, while a larger amount of finer particles could be seen in the microstructure of sample $S_{3}$. However, it should be noted that the partial presence of cobalt layer around the ceramic particles which could reduce the formation of detrimental products, increase microhardness, and form fingerprint-like intermetallic compounds compared to the $\mathrm{Mg}$ addition method. The cobalt coating method can therefore be seen to be a new useful method in these regards. The details of the magnesium addition method for the incorporation percent were previously published by Hashim et al. ${ }^{[11]}$

During the solidification of the composite, internal stresses are developed around the particles due to a difference in the CTE of the aluminum and the $\mathrm{SiC}$ particle, and they are relieved by formation and movement of dislocations. ${ }^{[30,32]}$ However, the formation of porosities would lead to crack initiation during local plastic deformation, leading to a reduction in hardness and other mechanical properties. In order to evaluate the effect of the magnesium and cobalt coating wetting aids on the mechanical properties of the fabricated samples, ten random points were selected within each sample for microhardness measurements (Vickers). Figure 10 shows the microhardness values of the samples.
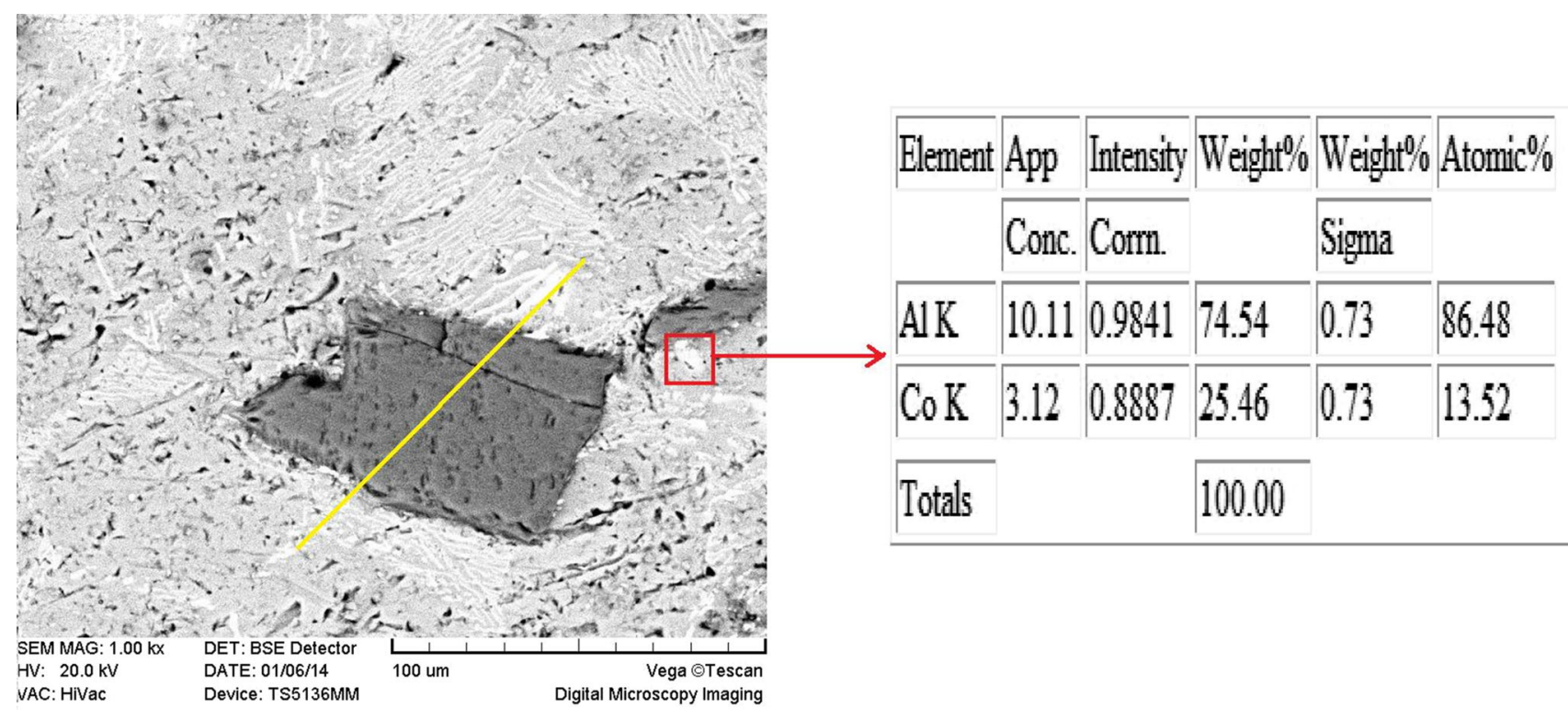

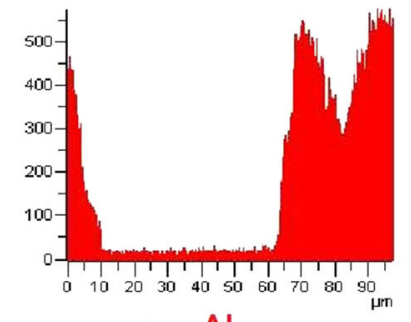

$\mathrm{Al}$

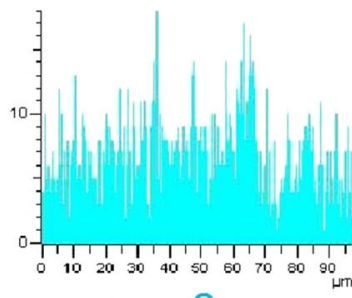

C

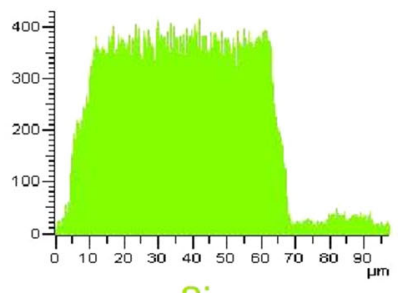

$\mathrm{Si}$

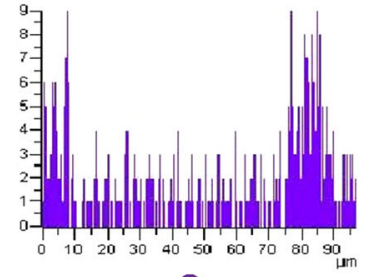

Co

Fig. 8- High-magnification SEM image of sample $\mathrm{S}_{3}$ as well as point and line EDAX analyses. 
0.35 vol. \%

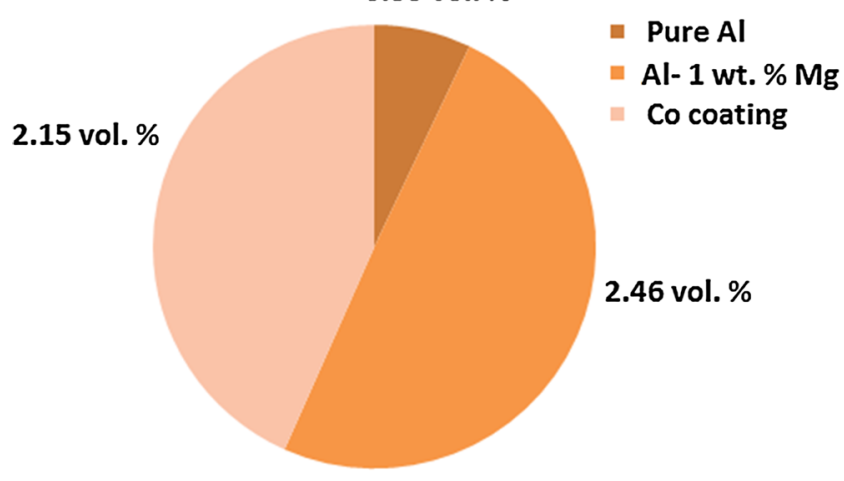

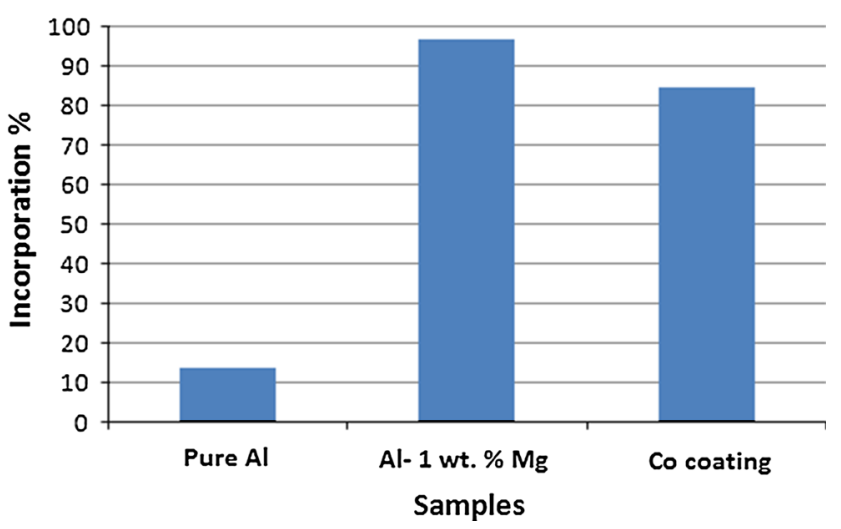

Fig. 9- The effect of metallic coating and $\mathrm{Mg}$ addition on the SiC particle incorporation percentage.

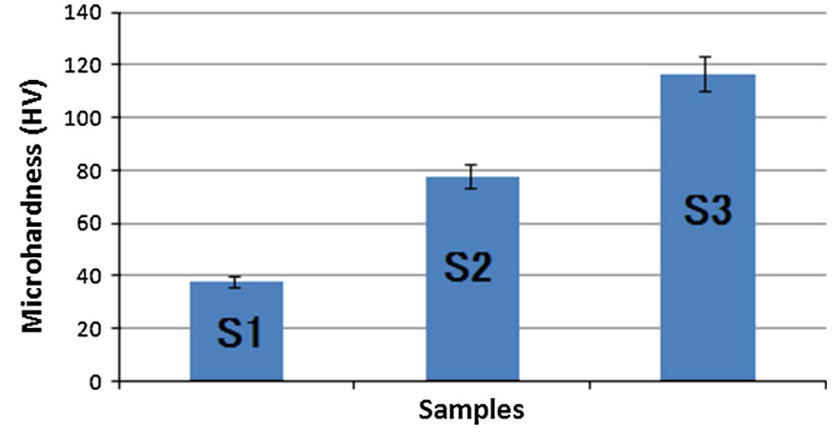

Fig. 10-Average values of Vickers microhardness test for casting samples.

The hardness of sample $S_{1}$ is very close to that of pure aluminum, meaning that the low incorporation of $\mathrm{SiC}$ particles (see Figure 4) did not highly affect to mechanical properties of the composite. However, the addition of magnesium, which led to increased incorporation of ceramic particles, also increased the hardness value by a factor of approximately two compared to that of sample $\mathrm{S}_{1}$. Although the ceramic incorporation percent of sample $S_{3}$ seems not to be much higher than that of sample $S_{2}$, the microhardness value recorded from this sample was much higher than (about 1.5 times) that of sample $S_{2}$. Reasons for the increased hardness in $S_{3}$ could include that the amount of ceramic particles incorporated was slightly higher than for sample $S_{2}$, a fingerprint-like structure of the Al-Co compounds was formed around the $\mathrm{SiC}$, smaller $\mathrm{SiC}$ particles were incorporated for sample $S_{3}$, and that a lower amount of agglomeration and porosity occurred within $\mathrm{S}_{3}$.

\section{CONCLUSIONS}

In this study, in order to increase the amount of ceramic particle incorporation into molten pure aluminum, cobalt coating was applied using the developed ED method. The results indicated that this method does provide improvement over alternative approaches and that it can be used with the stir casing process to incorporate high volumes of ceramic particulate into molten aluminum.
In particular, by comparing the microstructures of composites prepared with magnesium and cobalt to the sample produced without any wetting agent, both wetting agents were found to be very effective as regard ceramic incorporation. Cobalt metallic coating of $\mathrm{SiC}$ via $\mathrm{ED}$ is a more effective production route than the use of magnesium addition for incorporation of $\mathrm{SiC}$ within an aluminum matrix. This was observed via micrograph analysis in which less porosity, less agglomeration, and smaller particle size incorporation was observed within the cobalt-coated particulate-produced composites. The usage of the cobalt-coated $\mathrm{SiC}$ particles also produced a matrix composite with a higher amount of reinforcement compared to the usage of magnesium as the wetting. In addition, the microhardness test results indicated that a harder composite was fabricated using the cobalt-ED-coated $\mathrm{SiC}$ route in comparison with the magnesium wetting agent production route. The increased proportion of reinforcement, increased ceramicmatrix bonding strength, and formed aluminum-cobalt intermetallic phases are likely to be the main contributing factors to this beneficial increase in properties.

\section{REFERENCES}

1. N. Valibeygloo, R.A. Khosroshahi, and R.T. Mousavian: Int. J. Miner. Metall. Mater., 2013, vol. 20 (10), pp. 978-85.

2. M.R. Roshan, R.T. Mousavian, H. Ebrahimkhani, and A. Mosleh: J. Min. Metall. Sect. B, DOI:10.2298/JMMB120701032R.

3. S. Naher, D. Brabazon, and L. Looney: J. Mater. Process. Technol., 2004, vol. 166, pp. 430-39.

4. S. Naher, D. Brabazon, and L. Looney: J. Mater. Process. Technol., 2003, vols. 143-144, pp. 567-71.

5. S. Naher, D. Brabazon, and L. Looney: Composite A, 2007, vol. 38 , pp. $719-29$

6. R. RahmaniFard and F. Akhlaghi: J. Mater. Process. Technol., 2007, vols. 187-188, pp. 433-36.

7. S. Amirkhanlou and B. Niroumand: Mater. Des., 2011, vol. 32, pp. 1895-902.

8. F. Akhlaghi, A. Lajevardi, and H.M. Maghanaki: J. Mater. Process. Technol., 2004, vols. 155-156, pp. 1874-80.

9. D.J. Lloyd: Compos. Sci. Technol., 1989, vol. 35, pp. 159-79.

10. S. Tzamtzis, N.S. Barekar, N. HariBabu, J. Patel, B.K. Dhindaw, and Z. Fan: Composite A, 2009, vol. 40, pp. 144-51.

11. J. Hashim, L. Looney, and M.S.J. Hashmi: J. Mater. Process. Technol., 2001, vol. 119, pp. 329-35. 
12. A. Urena, E.E. Martinez, P. Rodrigo, and L. Gil: Compos. Sci. Technol., 2004, vol. 64, pp. 1843-54.

13. J. Hashim, L. Looney, and M.S.J. Hashmi: J. Mater. Process. Technol., 2001, vol. 119, pp. 324-28.

14. J. Hashim, L. Looney, and M.S.J. Hashmi: J. Mater. Process. Technol., 1999, vols. 92-93, pp. 1-7.

15. S.A. Sajjadi and H.R. Ezatpour: Mater. Des., 2012, vol. 34, pp. 106-11.

16. G.G. Sozhamannan, S.B. Prabu, and V.S.K. Venkatagalapathy: $J$. Surf. Eng. Mater. Adv. Technol., 2012, vol. 2, pp. 11-15.

17. A.E. Karantzalis, A. Lekatou, E. Georgatis, T. Tsiligiannis, and H. Mavros: JMEPEG, 2010, vol. 19, pp. 1268-75.

18. A. Canakci, F. Arslan, and T. Varol: Mater. Sci. Technol., 2013, vol. 29 , pp. $954-60$.

19. T. Varol and A. Canakci: Met. Mater. Int., 2013, vol. 19, pp. 1227-34.

20. T. Varol and A. Canakci: Powder Technol., 2013, vol. 246, pp. $462-72$.

21. T. Varol, A. Canakci, and S. Ozsahin: Composite B, 2013, vol. 54, pp. 224-33.

22. C.A. Leon, G. Mendoza-Suarez, and R.A.L. Drew: J. Mater. Sci., 2010, vol. 41, pp. 5081-87.
23. B.B. Singh and M. Balasubramanian: J. Mater. Process. Technol., 2009, vol. 209, pp. 2104-10.

24. A. Urena, J. Rams, M.D. Escalera, and M. Sanchez: Composite A, 2007, vol. 38, pp. 1947-56.

25. A. Urena, J. Rams, M. Campo, and M. Sanchez: Wear, 2009, vol. 266, pp. 1128-36.

26. C.Sh. Rames, R. Keshavamurthy, B.H. Channabasappa, and A. Ahmed: Mater. Sci. Eng. A, 2009, vol. 502, pp. 99-106.

27. F. Kretz, Z. Gacsi, J. Kovacs, and T. Pieczonka: Surf. Coat. Technol., 2004, vols. 180-181, pp. 575-79.

28. C.A. Leona and R.A.L. Drew: Composite A, 2002, vol. 33, pp. $1429-32$.

29. L.A. Dobrzański, M. Kremzer, and J. Konieczny: J. Achiev. Mater. Manuf. Eng., 2011, vol. 46 (2), pp. 147-53.

30. M. Mohammadpour, R.A. Khosroshahi, R.T. Mousavian, and D. Brabazon: Ceram. Int., 2014, vol. 40, pp. 8323-32.

31. A.J. McAIieter: Bull. Alloy Phase Diag., 1989, vol. 10 (6), pp. 64650.

32. P.K. Rohatgi, N. Gupta, and S. Alaraj: J. Compos. Mater., 2006, vol. 40 (13), pp. 1163-74. 